CERN-TH.7155/94

\title{
Two-Dimensional String Theory, Topological Field Theories and the Deformed Matrix Model
}

\author{
Ulf H. Danielsson \\ Theory Division, CERN, CH-1211 Geneva 23, Switzerland
}

\begin{abstract}
In this paper the $c=1$ string theory is studied from the point of view of topological field theories. Calculations are done for arbitrary genus. A change in the prescription is proposed, which reproduces the results of the $1 / x^{2}$ deformed matrix model. It is proposed that the deformed matrix model is related to a D-series Landau-Ginzburg superpotential.
\end{abstract}

CERN-TH.7155/94

January 1994 


\section{INTRODUCTION}

The tremendous success of the matrix models in describing two dimensional quantum gravity and low-dimensional string theory is in sharp contrast with the difficulties of the continuum approach. Recent developments connecting the $c=1$ model to an unorthodox Landau-Ginzburg model are beginning to change this. This promises to give new insights into the structure, and physics, of two-dimensional string theory.

Such an improved understanding is important for several reasons. The target space physics of the $c=1$ matrix model is very badly understood. It has been shown, see [1-6], that a deformation of the matrix model potential gives a new model, presumably also a model of a string moving in a twodimensional target space. It has been conjectured that this model is the black hole of ref. [7].

In this context it is important to come with some clarifications. In the work of [8] it has been suggested that the ordinary $c=1$ model can be thought of as describing a black hole. This is a consequence of the LandauGinzburg description and its association with the coset model

$$
\frac{S U(2)_{k=-3} \otimes[b, c]}{U(1)},
$$

which was derived in [8]. However, as pointed out in [8], this is not the black hole described by Witten, which instead is given by the coset

$$
\frac{S U(2)_{k=-9 / 4}}{U(1)} \otimes[b, c] .
$$

In this paper I will suggest a modification of the super-potential prescription for $c=1$, which reproduces the results of the deformed matrix model. The hope is that this will give further insight into the physics of the deformed matrix model and the target space physics of the two-dimensional string.

In Section 2 I will quickly go through the very basics of the connection between Landau-Ginzburg models and topological field theories. In Section 3 the techniques are generalized to $c=1$. Section 4 is devoted to higher genus, and Section 5 contains a comparison with more traditional $c=1$ matrix model calculations. Then, in Section 6, I propose a generalization of the construction, which is capable of describing the deformed matrix model. This is found to work also at higher genus. I also show that this implies that the deformed matrix model is associated with a D-series superpotential. 


\section{LANDAU-GINZBURG POTENTIALS AND GELFAND-DIKII EQUATIONS}

I will begin by recalling some of the basic building blocks in the description of topological string theory. In particular the relations between the $\mathrm{KdV}$ hierarchy and the Landau-Ginzburg potential.

By starting with the two-dimensional minimal $N=2$ superconformal field theories it is possible to obtain a sequence of topological field theories, see e.g. [9]. These $N=2$ models can be described by super potentials $W(X)$. The super potentials are classified according to the A-D-E scheme. For instance, one has the A-series with the potentials $W(X)=X^{k+2}$. They correspond to models with central charge $d=\frac{k}{k+2}$ with $k$ positive integer. These models can also be realized as $\frac{S U(2)_{k}}{U(1)}$ coset models, see [10. As shown in [9] there is also a direct relation between the super potentials and the KdV equations of the $c<1$ matrix models, which I will quickly go through below. A nice reference is [11], from where I will borrow some of the notation.

The crucial observation in [9] was that the superpotential, and its perturbations, can be directly identified with the KdV differential operator, i.e.

$$
W=D^{k+2}-\sum_{i=0}^{k} u_{i}(x) D^{i}
$$

in the case of the A-series. I have replaced $X$ with $D=\frac{i \hbar}{\sqrt{k+2}} \frac{\partial}{\partial x}$ and $x$ is the coupling of the puncture operator. The $u_{i}(x)$ are determined through the flow equations

$$
i \frac{\partial W}{\partial t_{m, n}}=\frac{(k+2)^{m+1 / 2}(-1)^{m}}{(n+1)(n+k+3) \ldots(n+1+m(k+2))}\left[W_{+}^{m+\frac{n+1}{k+2}}, W\right]
$$

with $t_{0}=x ; t_{m, n}$ is the coupling of $\sigma_{m}\left(\phi_{n}\right)$, i.e. the $m$ th descendant of the primary $\phi_{n}$. The +-index means keeping only positive powers of $D$. Restricting to the flow due to primaries one gets

$$
i \frac{\partial W}{\partial t_{n}}=\frac{(k+2)^{1 / 2}}{n+1}\left[W_{+}^{\frac{n+1}{k+2}}, W\right] .
$$

I have put $t_{n}=t_{0, n}$. The correlation functions of topological field theory are 
then defined through

$$
\left\langle\phi_{j}\right\rangle=\frac{\partial F}{\partial t_{j}}=\frac{(k+2)^{2}}{(j+1)(j+k+3)} \operatorname{res}\left(W^{1+\frac{j+1}{k+2}}\right),
$$

where 'res' picks out the coefficient of $D^{-1}$. Higher-order correlation functions are obtained by taking more derivatives and using the flow equations. One point functions of descendants are given by

$$
\left\langle\sigma_{m}\left(\phi_{j}\right)\right\rangle=\frac{(-1)^{m}(k+2)^{m+2}}{(j+1)(j+k+3) \ldots((m+1)(k+2)+j+1)} r e s\left(W^{1+m+\frac{j+1}{k+2}}\right) .
$$

Let me give an example of a correlation function. In the classical $(\hbar \rightarrow 0)$ limit where commutators are replaced by Poisson brackets, the three-point function for three primaries is given by

$$
\begin{gathered}
\frac{\partial^{3} F}{\partial t_{m_{1}} \partial t_{m_{2}} \partial t_{j}} \\
=\frac{(k+2)^{2}}{(j+1)(j+k+3)\left(m_{1}+1\right)\left(m_{2}+1\right)} \operatorname{res}\left(\left\{W_{+}^{\frac{m_{1}+1}{k+2}},\left\{W_{+}^{\frac{m_{2}+1}{k+2}}, W^{1+\frac{j+1}{k+2}}\right\}\right\}\right) \\
=\frac{k+2}{(j+1)\left(m_{1}+1\right)\left(m_{2}+1\right)} \operatorname{res}\left(\frac{\partial_{D} W_{+}^{\frac{m_{1}+1}{k+2}} \partial_{D} W_{+}^{\frac{m_{2}+1}{k+2}} \partial_{D} W^{\frac{j+1}{k+2}}}{\partial_{D} W}\right)
\end{gathered}
$$

I have used $\frac{\partial}{\partial x} W_{+}^{\frac{m+1}{r}}=0$, for $m \leq k$. At zero coupling $W_{+}^{\frac{m+1}{k+2}}=D^{m+1}$, this implies the standard result

$$
\frac{\partial^{3} F}{\partial t_{m_{1}} \partial t_{m_{2}} \partial t_{j}}=\delta_{m_{1}+m_{2}+j, k}
$$

Higher-point correlation functions are obtained analogously. This illustrates the connection between the KdV-operator the Landau-Ginzburg models. In the following I will often be careless and generally refer to $W$ as the superpotential, even when the argument is a differential operator $\frac{\partial}{\partial x}$. 


\section{THE $c=1$ MODEL FROM A $D^{-1}$ POTEN- TIAL}

In 12 it was found that if $k=-3$ then the topological theory computes the Euler characteristics of the moduli space of Riemann surfaces. Since this is also accomplished by the $c=1$ matrix model at the self dual radius $R=1$ (in units where $\alpha^{\prime}=1$ ), [13], it is reasonable to expect that this model is the $c=1$ string at $R=1$. In $[8]$ this conjecture was proven by an explicit construction of a twisted $N=2$ theory starting with $c=1$. The supercurrents were obtained by using the $b-c$ ghost system. Hence one is lead to consider models with superpotential $W=X^{-1}$.

In this and the next few sections I will illustrate the $k=-3$ case by some explicit examples and calculations, including higher genus. This is intended as a warm-up for Section 6 where, finally, I propose a way of extending the formalism to the case of the deformed matrix model.

Now, to understand how all of this works, we must adapt the formalism of the previous section to the case $D^{-1}$. This is not completely straightforward and several papers have been devoted to this subject [8, 14, 15].

I begin by specifying the superpotential $W$. I restrict myself to the small phase space, i.e. where all couplings to descendants are put to zero. The superpotential is then

$$
W=\mu D^{-1}-x+\sum_{i=2}^{\infty} t_{i} D^{i-1},
$$

where $D=-i \hbar \frac{\partial}{\partial x}$. I will sometimes keep the $\hbar$ for clarity but at the end it will always be put to 1 , or, more precisely, absorbed into $\mu$. It is important that $W$ is only linear in the couplings $t_{i}$. This implies that the primaries

$$
\phi_{m}=\frac{\partial W}{\partial t_{m}}=D^{m-1}
$$

do not flow.

The primaries $\phi_{m}$ are identified with tachyons, $T_{m}$, with positive momenta $m>0$. The negative-momentum tachyons, $T_{-m}$, correspond to descendants $\sigma_{m}\left(\phi_{0}\right)$ of the cosmological constant operator, i.e. $D^{-1}$. One might note that the puncture operator, in the sense of topological field theory, is the first special tachyon, $T_{1}$. This was first observed in [16]. 
The one-point function of a negative-momentum tachyon is

$$
\left\langle T_{-p}\right\rangle=\frac{1}{p(p+1)} \operatorname{res}\left(W^{p+1}\right) .
$$

From this, the expression for $W$ and the identification of the positive-momentum tachyons as the primaries (11), one can easily verify the matrix model results for the tachyon correlation functions. Indeed,

$$
\begin{gathered}
\frac{1}{p(p+1)} \operatorname{res}\left(\mu D^{-1}-x+\sum_{i=2}^{\infty} t_{i} D^{i-1}\right)^{p+1} \\
=\frac{1}{p(p+1)} \sum_{\sum_{i=1}^{l} m_{i} n_{i}=p} t_{n_{1}}^{m_{1}} \ldots t_{n_{l}}^{m_{l}} \mu^{p+1-\sum_{i=1}^{l} m_{i}} \frac{(p+1) p \ldots\left(p+2-\sum_{i=1}^{l} m_{i}\right)}{m_{1} ! \ldots m_{l} !} .
\end{gathered}
$$

Hence

$$
\left\langle T_{p_{1} \ldots} \ldots T_{p_{N-1}} T_{-p}\right\rangle=\frac{\partial}{\partial t_{p_{1}}} \ldots \frac{\partial}{\partial t_{p_{N-1}}}\left\langle T_{-p}\right\rangle=(p-1) \ldots(p-N+3) \mu^{p-N+2} .
$$

In [14, 15], correlation functions of more than one descendant, i.e. negativemomentum tachyon, are considered. This implies the presence of contact terms that need special care. I will not consider these complications. I will instead restrict myself to the simpler case where no contact terms are needed.

The main subject of the next section is to show that these calculations can be extended to higher genus. The claim is that if one remembers that $D=-i \hbar \frac{\partial}{\partial x}$, where $x$ is the coupling of the $T_{1}$ tachyon and if one defines the residue as the coefficient of $D^{-1}$ after that all the $x$ 's have been commuted to the left, then the higher genus results of the matrix model are reproduced.

Rather than proving this in the same way as above for genus zero, I will do it in a way that clearly illustrates the close connection with the matrix model. In fact, it will turn out that the calculations are formally identical to calculations of tachyon correlation functions done in [17-19]. I will use the property that

$$
\frac{\partial}{\partial t_{p}}=\frac{1}{i \hbar}\left[T_{p}, \cdot\right]
$$

to replace the $t_{p}$ derivatives with commutators. This will be the subject of the next section. 


\section{CORRELATION FUNCTIONS AT HIGHER GENUS}

\subsection{The Algebraic Structure}

Let me reconsider the calculation, in the previous section, of the tachyon correlation function, but now using relation (15). This implies

$$
\left\langle T_{k_{1}} \ldots T_{k_{N-1}} T_{-k_{N}}\right\rangle=\frac{(i \hbar)^{1-N}}{k_{1} \ldots k_{N}} \operatorname{res}\left(\left[T_{k_{1}},\left[T_{k_{2}},\left[\ldots\left[T_{k_{N-1}}, T_{-k_{N}}\right] \ldots\right]\right)\right.\right.
$$

where

$$
T_{k}=D^{k}
$$

and

$$
T_{-k}=\frac{1}{k+1}\left(\mu D^{-1}-x\right)^{k+1}
$$

for $k>0$. What is the algebra generated by the tachyons? On the sphere one finds, for the Poisson brackets,

$$
\left\{T_{k}, T_{-l}\right\}=k l T_{k-1} T_{-l+1}
$$

and in general

$$
\begin{aligned}
& \frac{1}{i \hbar}\left[T_{k}, T_{-l}\right]=\left\{T_{k}, T_{-l}\right\}_{M}=\frac{2 \mu}{\hbar} \sin \frac{\hbar}{2 \mu}\left(\frac{\partial}{\partial D_{2}} \frac{\partial}{\partial x_{1}}-\frac{\partial}{\partial D_{1}} \frac{\partial}{\partial x_{2}}\right) T_{k} T_{-l} \\
= & k l\left(T_{k-1} T_{-l+1}\right)_{W}+\frac{\hbar^{2}}{24 \mu^{2}} k(k-1)(k-2) l(l-1)(l-2)\left(T_{k-3} T_{-l+3}\right)_{W}+\ldots
\end{aligned}
$$

where $\{,\}_{M}$ denotes the Moyal bracket [20], and the subscript $W$ means Weyl ordering, (i.e. a sum over all possible orderings with equal weight). This is precisely the $W_{\infty}$ algebra of the $c=1$ matrix model. In the matrix model, see [21], the generators are

$$
W_{J, m}=(\lambda+p)^{J+m}(\lambda-p)^{J-m},
$$

obeying

$$
\left[W_{J_{1}, m_{1}}, W_{J_{2}, m_{2}}\right]=4\left(m_{2} J_{1}-m_{1} J_{2}\right) W_{J_{1}+J_{2}-1, m_{1}+m_{2}}+\ldots
$$


with $\hbar$ corrections given by the Moyal bracket if the operators are defined using Weyl-ordering, [18, 19, 22. So, we have seen that the algebraic structure of the model defined in the previous section is identical to that of the matrix model. This is true to for all genera. In the next section I will verify that the correspondence is also true for the one-point functions. The knowledge of the algebra and its higher genus corrections, together with the knowledge of the one-point functions, make it possible to calculate the correlation functions at arbritrary genus.

\subsection{The One-Point Functions}

In this section I will calculate the one-point functions

$$
\operatorname{res}\left(\left(D^{r}\left(\mu D^{-1}-x\right)^{r+1}\right)_{W}\right) \text {. }
$$

These correspond to one-point functions of $W_{r, 0}$. The residue is defined as the coefficient of the $D^{-1}$ term when all the $D$ 's have been commuted to the right.

I will need the formulae

$$
\left(q^{m} p^{n}\right)_{W}=2^{-m} \sum_{l=0}^{m}\left(\begin{array}{c}
m \\
l
\end{array}\right) q^{m-l} p^{n} q^{l}
$$

and

$$
\left[p^{n}, q^{m}\right]=\sum_{k=1}^{m i n(n, m)}\left(\begin{array}{c}
m \\
k
\end{array}\right)\left(\begin{array}{c}
n \\
k
\end{array}\right) k !(-i \hbar)^{k} q^{m-k} p^{n-k},
$$

given $[p, q]=-i \hbar$. A derivation of the first formula, given the definition of Weyl-ordering, can be found for instance in [23]. It is only valid for positive $n$ and $m$. In the second formula, however, one can allow either $m$ or $n$ to be negative. These combine to

$$
\left(q^{m} p^{n}\right)_{W}=\sum_{k=0}^{\min (n, m)} 2^{-k}\left(\begin{array}{c}
m \\
k
\end{array}\right)\left(\begin{array}{c}
n \\
k
\end{array}\right) k !(-i \hbar)^{k} q^{m-k} p^{n-k},
$$

valid only for $n$ and $m$ positive. Here $\sum_{l=0}^{n}\left(\begin{array}{c}n \\ l\end{array}\right)=2^{n}$ has been used. Since $D$ and $-\mu D^{-1}+x$ obey the same algebra as $p$ and $q$, it follows that

$$
\left(D^{r}\left(\mu D^{-1}-x\right)^{r+1}\right)_{W}
$$




$$
=\mu^{r+1} \sum_{k=0}^{r} 2^{-k}\left(\begin{array}{c}
r+1 \\
k
\end{array}\right) \frac{r !}{(r-k) !}\left(\frac{i}{\mu}\right)^{k}\left(\mu D^{-1}-x\right)^{r+1-k} D^{r-k} .
$$

Let me now commute $x$ to the left in the powers of $\mu D^{-1}-x$ and then put $x=0$. This gives

$$
\begin{gathered}
\mu D^{-1}-x \rightarrow \mu D^{-1} \\
\left(\mu D^{-1}-x\right)^{2} \rightarrow \mu D^{-1}\left(\mu D^{-1}-x\right) \rightarrow \mu^{2}\left(1+\frac{1}{i \mu}\right) D^{-2}
\end{gathered}
$$

and in general

$$
\left(\mu D^{-1}-x\right)^{k} \rightarrow\left(1+\frac{1}{i \mu}\right) \ldots\left(1+(k-1) \frac{1}{i \mu}\right) D^{-k}
$$

So the result is

$$
\begin{gathered}
\operatorname{res}\left(D^{r}\left(\mu D^{-1}-x\right)^{r+1}\right)_{W} \\
=\mu^{r+1} \sum_{k=0}^{r} 2^{-k}\left(\begin{array}{c}
r+1 \\
k
\end{array}\right) \frac{r !}{(r-k) !}\left(1+\frac{1}{i \mu}\right) \ldots\left(1+\frac{r-k}{i \mu}\right)\left(\frac{i}{\mu}\right)^{k} \\
=\sum_{k=0}^{r} 2^{-k}\left(\begin{array}{c}
r+1 \\
k
\end{array}\right) \frac{r !}{(r-k) !} i^{r-1}(-1)^{r-k}(i \mu)_{r-k+1} .
\end{gathered}
$$

where $(x)_{n}=x(x+1) \ldots(x+n-1)$. An expansion in genus is easily obtained using Stirling numbers of the first kind, $S_{n}^{(m)}$, as defined in 24]:

$$
x(x-1) \ldots(x-n+1)=\sum_{m=0}^{n} S_{n}^{(m)} x^{m} .
$$

We then get

$$
\sum_{k=0}^{r} 2^{-k}\left(\begin{array}{c}
r+1 \\
k
\end{array}\right) \frac{r !}{(r-k) !} i^{r+1} \sum_{m=0}^{r-k+1} S_{r-k+1}^{(m)}(-i \mu)^{m}
$$

The first few terms in the series are

$$
\mu^{r+1}\left(1-\frac{(r+1) r(r-1)}{12 \mu^{2}}+\frac{(r+1) r(r-1)(r-2)(r-3)}{5760 \mu^{4}}(20 r-8)+\ldots\right)
$$




\subsection{An Example}

To illustrate the above formulae, consider the two-point function $\left\langle T_{k} T_{-k}\right\rangle$ to genus one. First use the $W_{\infty}$ algebra of (20) to obtain

$$
\begin{gathered}
\left\langle T_{k} T_{-k}\right\rangle=\frac{1}{i \hbar k^{2}} \operatorname{res}\left(\left[T_{k}, T_{-k}\right]\right)=\operatorname{res}\left(D^{k-1} \frac{\left(\mu D^{-1}-x\right)^{k}}{k}\right)_{W} \\
+\frac{1}{24 \mu^{2}}(k-1)^{2}(k-2)^{2} \operatorname{res}\left(D^{k-3} \frac{\left(\mu D^{-1}-x\right)^{k-2}}{k-2}\right)_{W}+\ldots
\end{gathered}
$$

Then use the residue formula of the previous section to obtain the answer

$\mu^{k}\left(\frac{1}{k}-\frac{(k-1)(k-2)}{12 \mu^{2}}+\frac{(k-1)^{2}(k-2)}{24 \mu^{2}}\right)=\frac{\mu^{k}}{k}-(k-1)\left(k^{2}-k-2\right) \frac{\mu^{k-2}}{24}$

for the two-point to genus 1 . This agrees with the result of [25].

\section{A COMPARISON WITH THE MATRIX MODEL}

I will now compare the above calculational rules and results with what is obtained in the matrix model. I will use the techniques developed in [18, 19]. There it was shown how perturbation theory leads to the formula

$$
\left\langle P T_{k_{1}} \ldots T_{k_{N}}\right\rangle \sim\left\langle P\left[T_{k_{1}},\left[T_{k_{2}}, \ldots\left[T_{k_{N-1}}, T_{k_{N}}\right] \ldots\right]\right]\right\rangle
$$

up to the factorized external leg factors; $k_{1}$ through $k_{N-1}$ are positive while $k_{N}$ is negative. The right-hand-side two-point function is given by

$$
\langle P W\rangle=-\frac{1}{\pi} \mathcal{I} \sum_{n=0}^{\infty} \frac{\langle n\|W\| n\rangle}{E_{n}-\mu}
$$

$n$ labels the one-particle states in the matrix model harmonic potential and $E_{n}=\frac{2 n+1}{2 i}$ at $\alpha^{\prime}=1$. I have already, in eq. (20), written down the algebra and its higher genus $(\hbar)$ corrections. To complete the matrix model calculation it is necessary to calculate

$$
\left\langle\left(H^{r}\right)_{W} P\right\rangle .
$$


The easy object to calculate is

$$
\left\langle H^{r} P\right\rangle=\mu^{r} \log \mu \text {. }
$$

This follows immediately from the formal equality

$$
\sum_{n=0}^{\infty} \frac{f(n)}{n+z}=f(-z) \sum_{n=0}^{\infty} \frac{1}{n+z}
$$

if one only keeps the terms with a $\log \mu$. This is legitimate since, at a fixed positive power of $\mu$, these terms will dominate. In (38) $H=\frac{1}{2}\left(a a^{\dagger}+a^{\dagger} a\right)$ is Weyl-ordered. Henceforth I will drop all $\log \mu$ 's and external pole factors. My normalizations will be those of collective field theory.

Following [19] I will now calculate $\left(H^{r}\right)_{W}$. Consider

$$
\left(a^{r} a^{\dagger r}\right)_{W}=2^{-r} \sum_{l=0}^{r}\left(\begin{array}{c}
r \\
l
\end{array}\right) a^{\dagger r-l} a^{r} a^{\dagger l}
$$

Take the expectation value of this in the harmonic oscillator state $n$ :

$$
2^{-r} \sum_{l=0}^{r}\left(\begin{array}{l}
r \\
l
\end{array}\right)(n+l) \ldots(n+l-r+1) .
$$

Use $\langle n|H| n\rangle=n+\frac{1}{2}$ to deduce

$$
\left(a^{r} a^{\dagger r}\right)_{W}=2^{-r} \sum_{l=0}^{r}\left(\begin{array}{c}
r \\
l
\end{array}\right)\left(H-\frac{1}{2}+l\right) \ldots\left(H-\frac{1}{2}+l-r+1\right)
$$

and hence

$$
\left\langle\left(H^{r}\right)_{W} P\right\rangle=(2 i)^{-r} \sum_{l=0}^{r}\left(\begin{array}{c}
r \\
l
\end{array}\right)\left(i \mu-\frac{1}{2}+l\right) \ldots\left(i \mu-\frac{1}{2}+l-r+1\right) .
$$

This result is valid for the uncompactified case, i.e. $R=\infty$, to obtain the result for finite radius $R$, one should act with [25]:

$$
\frac{\frac{i}{R} \frac{\partial}{\partial \mu}}{e^{\frac{i}{2 R} \frac{\partial}{\partial \mu}}-e^{-\frac{i}{2 R} \frac{\partial}{\partial \mu}}}
$$


That is, at the self-dual radius $R=1$ :

$$
\left\langle\left(H^{r}\right)_{W}\right\rangle_{R=1}=\frac{i}{e^{\frac{i}{2} \frac{\partial}{\partial \mu}}-e^{-\frac{i}{2} \frac{\partial}{\partial \mu}}}\left\langle\left(H^{r}\right)_{W} P\right\rangle .
$$

I will now prove that, indeed,

$$
i\left\langle\left(H^{r}\right)_{W} P\right\rangle=\left(e^{\frac{i}{2} \frac{\partial}{\partial \mu}}-e^{-\frac{i}{2} \frac{\partial}{\partial \mu}}\right)\left\langle\left(H^{r}\right)_{W}\right\rangle_{R=1},
$$

where $\left\langle\left(H^{r}\right)_{W} P\right\rangle$ is given by (43) and $\left\langle\left(H^{r}\right)_{W}\right\rangle_{R=1}$ by

$$
\left\langle\left(H^{r}\right)_{W}\right\rangle_{R=1}=\frac{1}{r+1} \operatorname{res}\left(D^{r}\left(\mu D^{-1}-x\right)^{r+1}\right)_{W},
$$

where the right hand side is given by $(29)$. The necessary tools for this proof are the elementary formulae $e^{z \frac{\partial}{\partial x}} f(x)=f(x+z)$ and

$$
\left(x+\frac{1}{2}\right)_{n}-\left(x-\frac{1}{2}\right)_{n}=n\left(x+\frac{1}{2}\right)_{n-1} .
$$

The right-hand-side of (46) then becomes, using (29),

$$
-\frac{1}{r+1} \sum_{k=0}^{r} 2^{-k}\left(\begin{array}{c}
r+1 \\
k
\end{array}\right) \frac{r !}{(r-k) !}(r-k+1) i^{r-1}(-1)^{r-k}\left(i \mu+\frac{1}{2}\right)_{r-k} .
$$

A generalization of $(48)$

$$
\sum_{m=0}^{n}\left(\begin{array}{c}
n \\
m
\end{array}\right)(x-m)_{r}(-1)^{m}=r(r-1) \ldots(r-n+1)(x)_{r-n}
$$

transforms (49) into

$$
\frac{i^{r+1}}{r+1} \sum_{k=0}^{r} \sum_{m=0}^{k} 2^{-k}\left(\begin{array}{c}
r+1 \\
k
\end{array}\right)\left(\begin{array}{c}
k \\
m
\end{array}\right)(r-k+1)\left(i \mu+\frac{1}{2}-m\right)_{r}(-1)^{r-k+m} \text {. }
$$

Exchange the sums and use $\sum_{k=0}^{n}\left(\begin{array}{c}n \\ k\end{array}\right)(-2)^{-k}=2^{-n}$. The result of this is (43) and equality (47) is proven! This confirms that the approach of the previous section reproduces the results of the $c=1$ matrix model at $R=1$ for all genera. 


\section{THE DEFORMED MATRIX MODEL}

\subsection{Introduction}

I have now come to the case of the deformed matrix model. Let me quickly go through the basics of this model as it was introduced in [1] and developed in subsequent papers [2-6]. The deformed matrix model is obtained by adding a piece $M / 2 x^{2}$ to the matrix model potential. The potential becomes

$$
-\frac{1}{2 \alpha^{\prime}} x^{2}+\frac{M}{2 x^{2}}
$$

$M$ will be positive. The position of the Fermi level is still measured in terms of its deviation from zero, i.e. $\mu$. However, it is now possible to define a double scaling limit, even when $\mu=0$. One then needs to keep $\hbar / M^{1 / 2}$ fixed, which will be the string coupling constant.

Special cases of tachyon correlation functions have been calculated in several papers, $[2,3,5,6]$. In [6] the general formula (in the case with $N-1$ tachyons of the same chirality) up to genus one was calculated to be, for zero $\mu$,

$$
\begin{gathered}
\left\langle T_{\left.p_{1} \ldots T_{p_{N-1}} T_{-p}\right\rangle}\right. \\
=(N-3) ! ! p(p-2) \ldots(p-(N-4)) \prod_{i=1}^{N-1} p_{i}\left[M^{p / 2-N / 2+1}-(p-(N-2))\right. \\
\left.\times\left(\frac{p^{2}+(N-1) \sum_{i=1}^{N-1} p_{i}^{2}-2(N-2) p-4 N+1}{24}-\frac{(N-1)}{24 R^{2}}\right) M^{p / 2-N / 2}+\ldots\right],
\end{gathered}
$$

when normalized to collective field theory. At genus zero it is easy to use this formula to write down the tachyon $N$-point function also for non-zero $\mu$. In this case, not only $\hbar / M^{1 / 2}$ is kept constant in the double scaling limit, but also $\hbar / \mu$. Both are proportional to the string coupling constant. One finds

$$
\left\langle T_{p_{1} \ldots T_{p_{N-1}} T_{-p}}\right\rangle=\frac{1}{p} \frac{\partial^{N-2}}{\partial \mu^{N-2}}\left(M+\mu^{2}\right)^{p / 2} .
$$

\subsection{An Ansatz}

The purpose of this subsection is to reproduce the above results using a generalization of the framework exemplified in previous sections. The formula 
I will generalize to non-zero $M$ is not (12) but rather

$$
\left\langle T_{1} T_{-p}\right\rangle=\frac{\partial}{\partial x}\left\langle T_{-p}\right\rangle=\frac{-1}{p} \operatorname{res}\left(W^{p}\right) .
$$

I will propose that the following expression for $W$ should be put into (55):

$$
W=\left(M D^{-2}+\left(\mu D^{-1}-x+\sum_{i=2}^{\infty} t_{i} D^{i-1}\right)^{2}\right)^{1 / 2}
$$

At $M=0$ we get (10) back. Let me now verify that (56) gives the correct correlation functions on the sphere. In this case the ordering inside of (55) is trivial. After integration of (55) with respect to $x$ it is obtained that

$$
\left\langle T_{-p}\right\rangle=\frac{1}{p} \sum_{l=0}^{p / 2}\left(\begin{array}{c}
p / 2 \\
l
\end{array}\right) \frac{1}{p-2 l+1} M^{l} \operatorname{res}\left(D^{-2 l}\left(\mu D^{-1}-x+\sum_{i=2}^{\infty} t_{i} D^{i-1}\right)^{p-2 l+1}\right) \text {. }
$$

After derivations with respect to the parameters $t_{k}$, equation (54) is indeed obtained.

At this point some clarifications are needed. Throughout this section I will assume the following rule of integration

$$
\int d x\left(\mu D^{-1}-x+\sum_{i=2}^{\infty} t_{i} D^{i-1}\right)^{k}=\frac{-1}{k+1}\left(\mu D^{-1}-x+\sum_{i=2}^{\infty} t_{i} D^{i-1}\right)^{k+1} .
$$

The basic formula that defines correlation functions of topological gravity is often taken to be a two-point function of the form (55). The integration that leads to a one-point function of the form (12) is justified using the string equation, see e.g. [11. This is equivalent to the rule above.

Let me proceed to show that the agreement continues beyond the sphere. In this case, however, one must be careful with the ordering as determined by (56). $W^{p}$ is given, for even $p$, as an expansion in which all orderings of the factors $M D^{-2}$ and $\left(\mu D^{-1}-x+\sum_{i=2}^{\infty} t_{i} D^{i-1}\right)^{2}$ appear with equal weight. I will consider the two-point function to genus 1 . The only terms in (57) that will contribute are the ones with at most $x^{2}$ and just one power of $t_{m}$. Hence we should pick $p-2 l=2$, which gives

$$
\frac{1}{3 p} \sum_{r=0}^{p / 2-1} \operatorname{res}\left(\left(M D^{-2}\right)^{r}\left(\mu D^{-1}-x+\sum_{i=2}^{\infty} t_{i} D^{i-1}\right)^{3}\left(M D^{-2}\right)^{p / 2-1-r}\right) .
$$


We must then pick out the $x^{2}$ and $t_{m}$ terms, i.e.

$\left(-x+t_{m} D^{m-1}\right)^{3} \rightarrow 3 t_{m} x^{2} D^{m-1}+3 \hbar t_{m} x(m-1) D^{m-2}+\hbar^{2} t_{m}(m-1)(m-2)$,

and then make the substitution in (59). The $x$ and $x^{2}$ are commuted to the left, and put to zero. The term proportional to $\hbar^{2}$ is then

$$
\frac{1}{3 p} \sum_{r=0}^{p / 2-1} M^{p / 2-1} \operatorname{res}\left((6 r(2 r+1)-6 r(m-1)+(m-1)(m-2)) D^{m-p-1}\right) .
$$

This implies $m=p$, i.e. momentum conservation, and the final result is

$$
\frac{1}{p} M^{p / 2}-\frac{1}{12} M^{p / 2-1}(p-2)(p+2)
$$

if the genus zero term also is included. Let us now go back to the matrix model result (53). Specializing this formula to $N=2$ gives

$$
\frac{M^{p / 2}}{p}-\frac{1}{24}\left(2 p^{2}-7-\frac{1}{R^{2}}\right) M^{p / 2-1}+\ldots
$$

So, at $R=1$ the answer agrees with the result of the calculation based on (56)!

In the appendix, I give a similar calculation for the four-point function.

\subsection{A D-series Superpotential}

The $W$ of (56) looks very awkward, due to the square root. This can, however, be dealt with. In the deformed matrix model it is well known that only half of the discrete tachyon states appear, the ones with even momenta. This means, for instance, that the momentum $p$ in (55) is always even. Therefore it is natural to relabel all the states by replacing the primaries $\phi_{n}$ by $\phi_{n / 2}$ and descendants $\sigma_{m}$ by $\sigma_{m / 2}$. As a consequence it is rather the square of (56) that should be thought of as the generalized superpotential, i.e.

$$
W=M D^{-2}+\left(\mu D^{-1}-x+\sum_{i=2}^{\infty} t_{i} D^{i-1}\right)^{2}
$$

This still does look a bit strange. However, I will now show that this expression can be derived from a D-series superpotential. The D-series has 
been considered previously in the context of the traditional topological field theories [9, 26]. There the D-series potential

$$
X^{r}+\frac{1}{2} X Y^{2}
$$

was considered. I now claim that the deformed matrix model is based on the superpotential

$$
W=M X^{-1}-X Y^{2}+\sum_{i=0}^{\infty} 2 t_{2 i} Y X^{i} .
$$

Following [9, 26] I integrate out the $Y$ field (this is possible since it appears only quadratically) using its equation of motion

$$
Y=\sum_{i=0}^{\infty} t_{2 i} X^{i-1}
$$

There is also a Jacobian which is taken care of by the change of variables $X=Z^{2}$, see [9, 26]. The result of these manipulations is

$$
W=M Z^{-2}+\left(\sum_{i=0}^{\infty} t_{2 i} Z^{2 i-1}\right)^{2},
$$

which is (64) after the proper identifications! Hence I conclude that the deformed matrix model is given by a D-series Landau-Ginzburg potential $M X^{-1}-X Y^{2}$ with the deformations $Y X^{k}$. The $Y X^{k}$ are to be identified with the special tachyons $T_{2 k}$.

One should note that the coupling of the $T_{1}$ tachyon, $x$, is put to zero in (68). This is just as it should, since only even-momentum special tachyons exist in the deformed model. However, as is clear from the previous subsection, it still plays a crucial role in the higher genus calculations.

\section{CONCLUSIONS}

We have seen how the results of the matrix model can be obtained for all genera by using a topological field theory approach. I have also shown how

a change in the prescription is capable of reproducing the results of the deformed matrix model. 
It seems as if there are some rather remarkable connections between the matrix models and the Landau-Ginzburg formulation that is fully revealed only in the light of the deformed matrix model. The suggestive derivation of the deformed matrix model from a D-series superpotential should be a clue to the understanding of the physics behind the model. Perhaps this will teach us enough about target space string physics for us to finally understand the elusive matrix model black hole.

\section{Acknowledgements}

I would like to thank E. Verlinde and W. Lerche for discussions.

\section{Appendix}

In this appendix I will calculate the four-point correlation functions $\left\langle T_{m} T_{m} T_{m} T_{-p}\right\rangle$. The relevant terms of (55) are

$$
\begin{aligned}
& \frac{1}{p} \int d x \sum_{s=0}^{p / 2-2} \sum_{r=0}^{p / 2-2-s} \operatorname{res}\left(\left(M D^{-2}\right)^{r}\left(\mu D^{-1}-x+t_{m} D^{m-1}\right)^{2}\right. \\
& \left.\quad \times\left(M D^{-2}\right)^{s}\left(\mu D^{-1}-x+t_{m} D^{m-1}\right)^{2}\left(M D^{-2}\right)^{p / 2-2-r-s}\right) .
\end{aligned}
$$

Collect the factors of $\mu D^{-1}-x+t_{m} D^{m-1}$ and perform the $x$ integration to obtain

$$
\begin{gathered}
\frac{1}{p} \sum_{s=0}^{p / 2-2} M^{p / 2-2} \sum_{r=0}^{p / 2-2-s} r e s\left(\frac{1}{5} D^{-2 r}\left(\mu D^{-1}-x+t_{m} D^{m-1}\right)^{5} D^{-p+4+2 r}\right. \\
\quad-4 \hbar s \frac{1}{4} D^{-2 r}\left(\mu D^{-1}-x+t_{m} D^{m-1}\right)^{4} D^{-p+3+2 r} \\
\left.+\left(4 s^{2}+2 s\right) \hbar^{2} D^{-2 r}\left(\mu D^{-1}-x-t_{m} D^{m-1}\right)^{3} D^{-p+2+2 r}\right) .
\end{gathered}
$$

The first term becomes

$$
\frac{1}{5 p} M^{p / 2-2} t_{m}^{3} \sum_{s=0}^{p / 2-2} \sum_{r=0}^{p / 2-2-s} r e s\left(D ^ { - 2 r } \left(10 x^{2} D^{3 m-3}+30 \hbar x(m-1) D^{3 m-4}\right.\right.
$$




$$
\begin{gathered}
\left.\left.+\hbar^{2}(m-1)(25 m-35) D^{3 m-5}\right) D^{-p+4+2 r}\right) \\
=\frac{1}{p} M^{p / 2-2} t_{m}^{3} \sum_{s=0}^{p / 2-2} \sum_{r=0}^{p / 2-2-s}(4 r(2 r+1)-12 r(m-1)+(m-1)(5 m-7)) \delta_{3 m, p} \\
=M^{p / 2-2} t_{p / 3}^{3}(p-2)\left(\frac{p^{2}}{36}-\frac{p}{12}-\frac{1}{8}\right) .
\end{gathered}
$$

The second term becomes

$$
\begin{gathered}
-\frac{1}{p} M^{p / 2-2} t_{m}^{3} \sum_{s=0}^{p / 2-2} \sum_{r=0}^{p / 2-2-s} \operatorname{res}\left(\hbar s D^{-2 r}\left(4 x D^{3 m-3}+6 \hbar(m-1) D^{3 m-4}\right) D^{-p+3+2 r}\right) \\
=-\frac{1}{p} M^{p / 2-2} t_{m}^{3} \sum_{s=0}^{p / 2-2} \sum_{r=0}^{p / 2-2-s}(6 s(m-1)-8 r s) \delta_{3 m, p} \\
=-M^{p / 2-2} t_{p / 3}^{3} \frac{p}{48}(p-2)(p-4) .
\end{gathered}
$$

Finally, the third term is calculated to be

$$
M^{p / 2-2} t_{p / 3}^{3} \frac{p}{144}(p-2)(p-4) .
$$

Adding these contributions together, and taking the $t_{p / 3}$ derivatives, gives the answer

$$
M^{p / 2-2} \frac{p-2}{24}\left(2 p^{2}-4 p-18\right) .
$$

Quite remarkably, this agrees with (53) at $R=1$.

\section{References}

[1] A. Jevicki and T. Yoneya, Nucl. Phys B411 (1994) 64.

[2] U.H. Danielsson, Nucl. Phys. B410 (1993) 395.

[3] K. Demeterfi and J.P. Rodrigues, "States and Quantum Effects in the Collective Field Theory of a Deformed Matrix Model", Princeton preprint PUPT-1407, hepth/9306141.

[4] T. Eguchi, "c=1 Liouville Theory Perturbed by the Black Hole Mass Operator", Tokyo preprint UT 650, July 1993. 
[5] K. Demeterfi, I.R. Klebanov and J.P. Rodrigues, "The Exact S-matrix of the Deformed c=1 Matrix Model", Princeton preprint PUPT-1416, hepth/9308036.

[6] U.H. Danielsson, "The Deformed Matrix Model at Finite Radius and a New Duality Symmetry", CERN preprint CERN-TH.7021/93, hepth/9309157.

[7] E. Witten, Phys. Rev. D44 (1991) 314.

[8] S. Mukhi and C. Vafa, "Two Dimensional Black Hole as a Topological Coset Model of $c=1$ String Theory", Harvard preprint HUTP-93/A002.

[9] R. Dijkgraaf, E. Verlinde and H. Verlinde, Nucl. Phys. B352 (1990) 59, "Notes on topological string theory and 2-D quantum gravity", Based on lectures given at the Spring School on Strings and Quantum Gravity, Trieste, Italy, April 24 - May 2, 1990 and at the Cargèse Workshop on Random Surfaces, Quantum Gravity and Strings, Cargèse, France, May 28 - June 1, 1990 Trieste Spring School 1990:91-156.

[10] Z. Qiu, Phys. Lett B188 (1987) 207, A.B. Zamolodchokov and V.A. Fateev, Sov. Phys. JETP 90 (1986) 1553.

[11] E. Witten, "Algebraic Geometry Associated with Matrix Models of Two Dimensional Gravity", IASSNS-HEP-91/74.

[12] E. Witten, Nucl. Phys B371 (1992) 191.

[13] J. Distler, C. Vafa, Mod. Phys. Lett. A6 (1991) 259.

[14] A. Hanay, Y. Oz and R. Plesser, "Topological Landau-Ginzburg Formulation and Integrable Structure of 2d String Theory", IASSNS-HEP94/1, hep-th/9401030.

[15] D. Ghoshal and S. Mukhi, "Topological Landau-Ginzburg Model of Two-Dimensional String Theory", TIFR/TH/93-62, hep-th/9312189.

[16] Y. Kitazawa, "Puncture Operator in $c=1$ Liouville Gravity", TIT/HEP-181.

[17] U.H. Danielsson and D.J. Gross, Nucl. Phys. B366 (1991) 3. 
[18] U.H. Danielsson, Nucl. Phys. B380 (1992) 83.

[19] U.H. Danielsson, "A Study of Two-Dimensional String Theory", Ph.D. Thesis, Princeton, 1992, hepth/9205063.

[20] J.E. Moyal, Proc. Cambridge Philos. Soc. 45 (1949) 99.

[21] E. Witten, Nucl.Phys. B373 (1992) 187.

[22] S.R. Das, A. Dhar, G. Mandal and S.R. Wadia, Mod. Phys. Lett. A7 (1992) 937.

[23] T.D. Lee, "Particle Physics and Introduction to Field Theory", Harwood Academic Publishers 1981.

[24] M. Abramowitz and I.A. Stegun, "Handbook of Mathematical Functions", National Bureau of Standards, Washington, D.C., 1964.

[25] I.R. Klebanov and D. Lowe, Nucl. Phys. B363 (1991) 543.

[26] T. Eguchi, Y. Yamada and S.K. Yang, Mod. Phys Lett. A8 (1993) 1627. 